\title{
Infección extra e intrahospitalaria por virus sincicial respiratorio en lactantes
}

\author{
María Angélica Palomino M. ; Carmen Larrañaga L. 2; Gustavo Montaldo L. 2; \\ Mónica Suárez G. ${ }^{2}$; Armando Díaz C. ; Luis Fidel Avandaño C. ${ }^{2}$
}

\section{Respiratory syncytial virus infection in infants and children}

\begin{abstract}
Clinical aspects of respiratory syncytial vicus (RSV) infection were sludied in 131 infants under age two years who were admited to hospilal for acute lower respiratory lroct infection [ALRI) during the cold seasons of year 1988 at Sanliago, Chile. Two daly samples of nasopharyngeal ospirates for RSV inmunofluorescent assay and viral isolation in Hep 2 cell culture, blood cells count and chest $X$ ray were performed at admission. Fifly three of these pratients showed positive immunofluorescense for $8 S V$ al admission. Nosophoryngeol somples were also prospeclively token to 251 inlonls admited to wwo hospital rooms al the cold seasons of years 1988 throughout 1989, at admission for virol isolation and immunofuorescent assoy and in olternale days for this late assay, along their hospital stays. Nasophanyngeol samples ablained after admission allowed delection of 42 coses of RSV nosxomial infections. No differences were found in clinical, hematological and rodiological features among palients with and without RSV intections. Nosocamial RSV inlection rale was $16,7 \%$ and $76 \%$ of cases occurred in the first week atler admission. Thal rale was not relaled to inlensily of RSV conlacts neither lenght of hospital slay. Since there ore no known specific differential features belween viral and bacterial respiratory infeclion, rapid methods for viral identification ore highly recommended for better manogement of acute lowes respirolory troct infections.
\end{abstract}

(Koy words: respiratory syncytiol viruses, virus diseases, respiralory tract infections, infant.)

Las infecciones respiratorias agudas bajas (IRAB) son la causa más frecuente de hospitalización en lactantes, especialmente en la estaciones frías, y de muerte de niños entre un mes y cuatro anos de edad ${ }^{1-3}$. En Chile los virus respiratorios son la primera causa de IRAB en lactantes $y$, entre ellos, el virus respiratorio sincicial (VRS) ocupa un rol preponderante, detectándose con frecuencias de $42 \%$ a $70 \%$, dependiendo de la forma de estudio ${ }^{4-9}$. Estos hallazgos son semejantes a los comunicados en ouros países ${ }^{10,1 t}$.

La posibilidad de realizar un diagnóstico etiológico rápido ha aumentado al disponer de técnicas de inmunofluorescencia ${ }^{5,799,12,13}$. La detención de VRS en niffos sanos es poco frecuente,

1. Servicio y Departamento de Pediatría, Hospital Roberto del Río, SS Metropolitano Norte, Facultad de Medicina None, Universidad de Chile.

2. Departamento de Microbiología, Facultad de Mcdicina Norte, Universidad de Chile.

Trabajo financiado pascialmente por Proyecto Fondecyı $097 / 88$. por lo que su hallazgo podría implicar un rol patogénico ${ }^{5,14}$. La mayoría de las infecciones por VRS son de carácter leve, pero algunas comprometen el tracto respiratorio inferior, produciendo bronconeumonías o bronquiolitis que requicren hospitalización; además, el VRS causa frecuentemente infecciones nosocomiales ${ }^{7}$. La presentación de la infección por VRS en forma epidémica todos los inviernos, unida a una semiología clínica característica, podría permitir al pediatra sospechar esta etiología sin necesidad de recurrir a exámenes virológicos, todavía poco accesibles.

Este trabajo se hizo con el propósito de describir los aspectos clínicos, radiológicos y de laboratorio general de los lactantes hospitalizados por IRAB asociada a VRS y algunos aspectos epidemiológicos de la infección nosocomial por VRS.

\section{Material y Método}

Estudio clínico. Durante $\mathrm{el}$ inviemo de 1988 (junioacubre) se estudiaron 131 niños menores de dos años in- 
gresados por IRAB comprobadas radiológicamentc, a dos salas de lactantes del Hospital Roberto del Rio, Santiago, Chile, destinadas preferentemente a enfermedades respiratorias. A todos ellos se les tomaron dos muestras de aspirado nasofaríngea en los primeros dos días de hospitalización para detección de VRS por inmunofluorescencia indirecta (IFI) y aislamiento en células Hep-2 $y$, además, hemograma, velocidad horaria de sedimentacíón de los erírocitos (VHS) y radiografía de tórax al ingresar. A cada niño se le confeccionó una ficha -hecha con ese propósito-, en la que se registraron edad, estado de la nutrición, síntomas previos, hechos dcl examen físico de ingreso, antecedentes personales, familiares y socioeconómicos.

Casos nosocomiales. En forma prospectiva se bizo seguimicnto de dos salas de hospitalización de un servicio de lactantes, dedicadas preferentemente a enfermedades respiratorias, del mismo hospital, ducante las temporadas frías de 1988 y 1989. Se tomó muestra de aspirado nasofaríngeo al ingreso para IFI y aislamiento viral, y sc continuó tomándolas día por medio -para IFI- durante toda la hospitalización: en los casos positivos se recolectó una muestra diaria, hasta obtener tres resultados consecutivos negativos. Los casos nosocomiales se detectaron confeccionando un registro de cada sala en seguimiento, consignando cada día el número de casos positivos. Para cuantificar la exposición al VRS a que estuvieron sometidos los lactantes hospitalizados en deterninada sala, se definió como contacto simple día niño a la presencia en la sala de ur caso climinador de virus durante un día; si existieron dos o tres casos excretando VRS simuliáneamente en la sala, se consideraron como contactos dobles o triples. Los casos positivos se clasificaron en ingresos o nosocómiales, para lo cual se definió como infección intrahospitalaria a aquellos pacicntes que climinaron virus a pariir del cuano día de hospitalización, tcniendo las mucstras de ingreso negativas. Fl análisis clínico se hizo registrando diariamente los signos y síntomas de cada caso nosocomial diagnosticado. La tasa de infección nosocomial durante el período estudiado se calculó considerando el mímero de casos que adquiricron el virus durante la hospitalización en relación a los casos ingresados sin VRS. E1 análisis de los factores de riesgo de contagio nosocomial se hizo considerando la magnitud del contacto con VRS y de la duración de la hospitalización.

Fstudio virolágico. Se consideraron posilivos los casos diagnosticados por IFI o cultivo confirmado para VRS ${ }^{12}$. La muestra de aspirado nasofaríngeo se transportó en bielo al laboratorio antes de una hora de recolectada y se hizo inoculación en Hep-2 o cnsayo de IFI o ambos mediante los procedimientos habiuales ${ }^{12}$, ${ }^{13}$. La IFI se efectuó con anticuerpos monoclonales anti VRS-proporcionados por el Dr. L. Anderson, CDC, Atlanta, GA-y conjugado cotnercial (SIGMA). Se cunsideraron posílivos los casos que mosttahan, al microscopio de luz UV, al menos tres células epíteliales con fluorescencia verdosa intensa en el ciloplasma. Para aislamiento sc inoculo $0,1 \mathrm{ml}$ del sobrenadante de la muestra centrifugada a I $500 \mathrm{rpm}$ por 20 min y se observó la aparición de efecto citopático (ECP) día por modjo dorante 14 días; en caso de ECP sospechoso y al final de 14 días de observación se realizo IFI del cullivo para confirmar el diagnóstico.

Para el cálculo de la significación estadística de las frecuencias observadas se vilizaron los mélodos de chi cuadrado y cálculo del error típico de la dilerencia de proporciones ( $($ ) asignando diferencia significativa al nivel de $5 \%$

\section{Resultados}

Estudio clínico. Se incluyeron 131 Jactantes ingresados por infección respiratoria aguda baja comprobada radiológicamente, 53 tenían JFI posiliva para VRS al ingreso y en 78 la reac. ción fue negativa durante toda la hospitalización.

El promedio de dias de hospitalización de los niños con VRS al ingreso fue 8,1 (márgenes 2 a 28) días y en los que no tenían VRS 9,25 (márgenes 2 a 36) días. La mayoría de los pacientes eran eutróficos $(75,5 \%)$. La edad, en $64,1 \%$ de los lactantes con y 61,5\% sin VRS, era menos de 6 mescs, siendo el promedio de aquélla 5,5 y 6 meses respectivamente. No se registraron muertes en los casos estudiados.

Los signos clínicos y los hallazgos rađiológicos al ingreso se muestran en la tabla 1. La mayorfa de los pacientes cursaron con temperatura sobre $38,4^{\circ} \mathrm{C}$, tanto con $(75,4 \%)$ o sin VRS $(74,3 \%)$. En más de la mitad la frecuencia respiratoria era más de 40 por min, no observándose diferencias entre los enfemos con y sin VRS. Sc registraron signos de obstrucción bronquial en $69,8 \%$ de los laclantes VRS positivo y en $55,1 \%$ de los VRS negativo. Se detectaron crépitos en $75,4 \%$ de los pacientes con y $71,7 \%$ de los sin VRS. Estas diferencias no son estadísticamente significativas. En las radiografías de tórax no se observó un patrón característico en los casos VRS positivos. La leucocitosis ( $\geq 15000$ lcucocitos por $\mathrm{mm}^{3}$ ) y la desviación a izquierda $(\geq 500$ baciliformes por $\left.\mathrm{mm}^{3}\right)$, no mostraron diferencias significativas. (Tabla 2). Los promedios de VHS fueron 25 y $26 \mathrm{~mm} / \mathrm{h}$ para los casos con y sin VRS respectivamentc, diferencias que tampoco son significativas.

Infección nosocomial. La tasa de infección nosocomial durante el período estudiado, considerando 42 casos que adquiricron el virus durante la hospitalización y 251 casos ingresados sin VRS, fuc de $16,7 \%$. En $54,2 \%$ de los casos nosocomiales la situación nutricional era de eutrofia, $31,4 \%$ eran desnutridos leves y $14,4 \%$ desnutridos avanzados. La frecuencia de casos nosocomiales por edad fuc: $16 / 83(19,3 \%)$ menores de 3 meses. $10 / 54(18,5 \%)$ cnirc 4 y 6 meses y $16 / 114$ (14\%) de 7 meses y más. Hubo predominancia del sexo masculino, tanto en los lactantes ingrcsados sin VRS (62\%) como en los casos nosocomiales (60\%). 
Tabla 1

Signos clínicos y radiológicos de 131 lactantes menores de dos años hospitalizados por IRA. Distribución según detección de VRS (junio a octubre de 1988)

\begin{tabular}{|c|c|c|c|c|c|c|}
\hline & \multicolumn{2}{|c|}{ VRS + } & \multicolumn{2}{|c|}{ VRS - } & \multirow[t]{2}{*}{$\mathbf{t}$} & \multirow[t]{2}{*}{$\mathbf{p}$} \\
\hline & n (53) & \% & $n(78)$ & $\%$ & & \\
\hline \multicolumn{7}{|l|}{ Signos elínicos } \\
\hline $\begin{array}{l}\text { Temperatura sobre } 38,4^{\circ} \mathrm{C} \\
\text { Frecuencia respiratoria sobre } 40 \\
\text { Obstrucción bronquial * } \\
\text { Crépitos }\end{array}$ & $\begin{array}{l}40 \\
31 \\
37 \\
41\end{array}$ & $\begin{array}{l}75,4 \\
58,4 \\
69,8 \\
75,4\end{array}$ & $\begin{array}{l}58 \\
48 \\
43 \\
56\end{array}$ & $\begin{array}{l}74,3 \\
61,5 \\
55,1 \\
71,7\end{array}$ & $\begin{array}{l}0,142 \\
0,356 \\
1,750 \\
0,470\end{array}$ & $\begin{array}{l}>0,80 \\
>0,70 \\
>0,05 \\
>0,60\end{array}$ \\
\hline \multicolumn{7}{|l|}{ Slgnos radiologlcos } \\
\hline $\begin{array}{l}\text { Hiperinsuflacion } \\
\text { Intersticial } \\
\text { Alveolar } \\
\text { Atelectasia } \\
\text { Otros }\end{array}$ & $\begin{array}{r}35 \\
44 \\
32 \\
5 \\
0\end{array}$ & $\begin{array}{r}66,0 \\
83,0 \\
60,0 \\
9,0 \\
0,0\end{array}$ & $\begin{array}{l}44 \\
66 \\
45 \\
9 \\
3 * *\end{array}$ & $\begin{array}{r}56,0 \\
85,0 \\
58,0 \\
12,0 \\
4,0\end{array}$ & $\begin{array}{l}1,164 \\
0,307 \\
0,229 \\
0,558\end{array}$ & $\begin{array}{l}>0,20 \\
>0,70 \\
>0,80 \\
>0,50\end{array}$ \\
\hline
\end{tabular}

- Sibilancias, retracción, espiración prolongada

** 2 derrame pleural, 1 neumatocele

\section{Tabla 2}

Algunos índices hematológicos de ingreso de 118 lactantes menores de dos años hospitalizados por IRAB. Distribución según detección de VRS (junio a octubre de 1988)

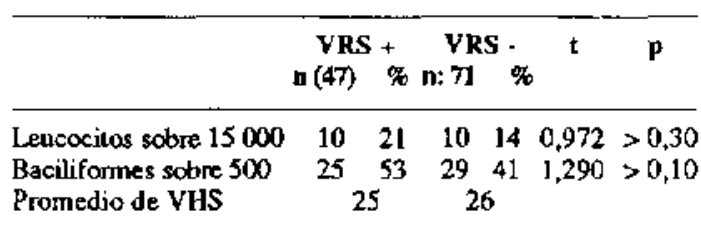

En 13 casos no se consiguió hemograma

El promedio de contactos simples (dí/niño) fue mayor para el grupo que adquirió la infección nosocomial $(6,45)$ que en los que no se infectaron con VRS $(4,62)$, lo mismo sucedió con los contactos dobles, pero en ambos casos las diferencias no fueron significativas (tabla 3). Los casos nosocomiales tuvieron un promedio de contactos con 4,69 niños excretores de VRS hasta el momento del diagnóstico de la infección intrahospitalaria, mientras, en los que no se infectaron, éste ocurrió con 2,48 casos positivos durante toda su hospitalización, diferencia estadisticamente significativa (t: 3,$70 ; \mathbf{p}<0,001$ ). Los casos nosocomiales tuvieron un promedio de 1,03 contactos/día en los 10 días previos al inicio de la excreción viral.
El promedio de días de estada fue de 11,16 dias (DE 7,35) para los casos nosocomiales $y$ 8,93 días (DE 11,66 ) para los casos sin VRS de ingreso, diferencia no estadísticamentc significativa ( $\mathrm{t}: 1,60 ; \mathrm{p}>0,1$ ). El promedio de estadía previa a la infección nosocomial fue de 6,8 días; $76,2 \%$ de los casos nosocomiales se detectaron durante la primera semana y $23,8 \%$ después de los 7 días. La duración de la excreción viral varió entre 1 y 14 días con promedio de 2 días.

\section{Comentario}

Es difícil hacer un diagnóstico diferencial entre IRAB de etiología viral o bacteriana basándose en las características clínicas y de laboratorio general. Inicialmente se pensó que las IRABs eran predominantemente de causa bacteriana y que $S$, pneumoniae y $H$. influenzae ocupaban un lugar preponderante. Posteriormente, con el desarrollo de técnicas rápidas de diagnóstico viral, se pudo demostrar que la mayoría de ellas tenían etiología viral. Diversas limitaciones dificultar la demostración de la etiología bacteriana: la flora bacteriana normal en el árbol respiratorio superior que contamina las muestras, el bajo rendimiento de los hemocultivos y del inmunodiagnóstico de antígenos en sangre y orina, las limitaciones éticas del uso de métodos invasivos más específicos, como la punción pulmonar. Por el 


\section{Tabla 3}

Infección intrahospitalaria por VRS en lactantes. Distribución según número y tipo de contactos (junio a noviembre 1988 y 1989)

\begin{tabular}{|c|c|c|c|c|c|c|}
\hline & \multicolumn{2}{|c|}{$\begin{array}{l}\text { Nosocomiales } \\
\text { (n: 42) }\end{array}$} & \multicolumn{3}{|c|}{$\begin{array}{c}\text { VRS negalivos } \\
\text { (n: 209) }\end{array}$} & \multirow[b]{2}{*}{$\mathbf{p}$} \\
\hline & $\overline{\mathbf{x}}$ & DE & $\overline{\mathbf{x}}$ & $\mathrm{DE}$ & $\mathbf{t}$ & \\
\hline \multicolumn{7}{|l|}{$\begin{array}{l}\text { Contactos } \\
\text { día/niño }\end{array}$} \\
\hline $\begin{array}{l}\text { Simples (total) } \\
\text { Dobles } \\
\text { Triples }\end{array}$ & $\begin{array}{l}6,45 \\
1,07 \\
0,71\end{array}$ & $\begin{array}{l}6,65 \\
1,44 \\
1,25\end{array}$ & $\begin{array}{l}4,62 \\
0,78 \\
0,41\end{array}$ & $\begin{array}{l}4,73 \\
1,18 \\
0,69\end{array}$ & $\begin{array}{l}1,697 \\
1,220 \\
1,510\end{array}$ & $\begin{array}{l}>0,05 \\
>0,20 \\
>0,10\end{array}$ \\
\hline
\end{tabular}

contrario, como no existe una flora viral normal en el aparato respiratorio, la detección de un virus implica una infección aguda que podría ser responsable del cuadro clínico; sin embargo, no podría atribuirsc siempre la enfermedad al virus detectado en el aspirado nasofaríngeo, pues puede haber inlecciones subclinicas ${ }^{14}$.

Diversos estudios a lo largo del país han destacado el rol del VRS como agente etiológico protagónico en las IR AB del lactante. Estos trabajos no han demostrado una asociación clara cntre manifestaciones clínicas y etiologia vira! específica, especialmente cuando se analizan los diagnósticos de ingreso (bronconeumonía, bronconeumonía con síndrome bronquial obstructivo, síndrome bronquial obstructivo) de los grupos con o sin detección de VRS ${ }^{4-9}$.

En este trabajo no se observó relación entre grado de desnutrición e infección por VRS y la distribución por edad mostró una concentración bajo los 6 meses tanto en los casos VRS positiyos como negativos. Al hospital ingresan normalmente niños seleccionados por factores de riesgo para muchas afecciones, entre ellos edad menor de 6 meses. Por lo tanto, no podemos concluir que el VRS afecta preponderantemente a este grupo de edades, pues dentro del espectro de severidad clínica de la infección por VRS sólo se hospitaliza una minoría, constituida por los niños más pequeños y graves, mientras que la mayoria sufre una enfermedad leve manejable en forma ambulatoria ${ }^{14}$.

El análisis de algunos indicadores clínicos confirma que, en comparación con los controles VRS negativos, no hay un patrón característico de infección respiratoria aguda baja por VRS desde un punto de vista clínico, radiológico y de laboratorio generaj!s. 7. ${ }^{25}$. Clásicamente se describían como signos orientadores de infección bacleriana los crépitos, la fiebre y la auscncia de sibilancias ${ }^{2}$. En nuestra scrie no observamos diferencias en la frecuencia de tiebre y crépitos entre los casos con o sin VRS. La obstrucción bronquial se observó con mayor frecuencia en los pacientes VRS positivos, pero la diferencia no es significativa. La radiología de tórax tampoco mostró predoninancia de hiperinsuflación ni imágenes intersticiales en los casos con VRS, como se había descrito clásicamente ${ }^{16}$. Lo mismo ocurrió con el hemograma, que no confirmó la VHS baja o ausencia de leucocitosis y de desviación a izquierda. Como forma de medir la gravedad, la duración de la hospitalización tampoco fue mayor en los casos VRS positivo.

En los pacientes que adquirieron la infección en el hospital, la mitad no presentó cambios de los síntomas y la mayoría sólo mostró síntomas respiratorios altos, no obstante que ellos ya sufrían una cnfermedad suficientemente severa como para motivar una hospitalización?. El período de hospitalización similar en los casos nosocomiales y los VRS ncgativos y la aparición de la mayoría de las infecciones hospitalarias en la primera semana, implica que la prolongación de la hospitalización no fue un factor favorecedor de infección nosocomial. Por su parte, los niffos que no se contagiaron estuvieron en el hospital 8,9 días, esto es, más que los 6,8 días que tardaron los infectados en el hospital en adquirir la infección y además tuvicron contacto con casos excretores de VRS, teniendo, por lo tanto, suficientes oportunidades para contagiarse.

En base a un mapa confeccionado para cada sala se buscó una relación entre la magnitud del contacto con VRS y la frecuencia de infección secundaria, observándose una tendencia a que los niños con mayor exposición al virus adquirieran la infección, lo que fue estadísticamente significativo cuando se analizó el número de niflos con VRS que estuvicron en contacto con los casos nosocomiales ${ }^{1 ?}$. La distribución de casos hospitalarios fue similar en edades menores de 3 meses y entre 4 y 6 meses, lo que hace suponer, por un lado, que no hay protección de anticuerpos maternos y, por otro, que éstos no favorecian la aparición de síntomas de acuerdo a la tesis de la reacción ínmunoalérgica entre anticuerpos matcrnos fijos en el pulmón y el virus"'. Otro factor que podria influir en el contagio intrahospitalario 
es el antecedente de infección previa por VRS. Si bien la infección por VRS puede repetirse y las reinfecciones tienden a ser menos sintomáticas, no está claro qué građo de inmunidad deja. En lactantes es muy dificil determinar la existencia de infecciones anteriores por VRS, pues la serología es poco útil. En efecto, hay transmisión pasiva de anticuerpos matemos que puede interferir en el diagnóstico, la respuesta inmune de los lactantes ante la infección por VRS en inconstante y la tecnologla disponible, que requiere de dos muestras de suero, es difícil de implementar ${ }^{11}$.

La duración de la excreción viral de los casos nosocomiales fue de dos días, la que podría estar distorsionada, porque varios niños se dieron alta antes de negativizarse la IFI.

En conclusión, no existe un patrón clínicoradiológico patognomónico de infección respiratoria aguda baja por VRS. La infección nosocomial por VRS se adquiere precozmente; su frecuencia no aumenta en forma importante al prolongar la estadía hospitalaria y no es proporcional a la magnitud del contacto. La alta frecuencia de la infección por VRS y la benignidad de la infección nosocomial no harian recomendable el aislamiento de cada caso VRS positivo, el que podría restringirse a pacientes de alto riesgo, como prematuros, lactantes portadores de displasia broncopulmonar, inmunodeprimidos, cardiópatas severos y daño neurológico ${ }^{18,19}$. Finalmente, el uso de técnicas rápidas de diagnóstico viral en un servicio destinado a infecciones respiratorias podría permití un enfoque racional de la IRAB viral y evitar terapias antibióticas innecesarias.

\section{Resumen}

Con el propósito de describir los aspectos clínicos de la infección por VRS se estudiaron 13] lactantes menores de dos afios hospitalizados por infección respiratoria aguda baja en los inviernos de 1988 y 1989 en Santiago. Al ingreso se les hizo aspirado nasofaríngeo para inmunofluorescencia anti-VRS, hemograma, velocidad horaria de eritrosedimentación, radiografía de tórax y se consignaron los signos clínicos. Cincuenta y tres de ellos tenían infección por VRS. La recolección de muestras de aspirado nasofaríngeo día por medio en dos salas de hospitalización permitió detectar 42 casos nosocomiales entre 251 ingre. sos no infectados originalmente. No se encontraron diferencias en los signos clínicos, hematológicos y radiológicos de ingreso entre casos con o sin VRS. La tasa de infección nosocomial fue $16,7 \%$; en $76 \%$ de estos casos ella se adquirió durante la primera semana, su frecuencia no fue proporcional a la magnilud del contacto con lactantes excretores de VRS y los afectados no tuvieron mayor estadía hospitalaria que los restantes pacientes. La ausencia de signos diferenciales entre infecciones respiratorias bajas virales y bacterianas hace resaltar la utilidad de los métodos rápidos de diagnóstico para virus en el manejo racional de estas afecciones.

(Palabras clave: virus respiratorio sincicial, infecciones respiratorias bajas, lactantes).

\section{Referencias}

1. Avendaña LF: Resfróo común, influenza y otras infeccjones víricas. En: Mencgucllo J, Fanta E, Paris E, Rosselot J, eds. Pediatria. Santiago: Mediterráneo, 1991: 879-883.

2. Toro $J$ : Mortalidad por infecciones respiratorias agudas en Chile. Pediatría (Santiago) 1986; 29:65-67.

3. Koempfer AM, Medino $E$ : llospitalizacion infantil en Chile: situación actual y perspectivas. Rev Chil Pediatr 1992; 63: 110-117.

4. Vicente $M, W_{\Lambda} E$, Carrasco $L_{r}$ el al.: Panicipación viral en las IRAB del lactante. Rev Chil Pediats 1988; 59; 530-535.

5. Ceruti $E$, Díaz $A$, Vicente $M$, et al.: Etiología de las infecciones respiratorias bajas agudas en lactantes hospitalizados. Rev Chil Pediatt 1991; 62: 155.166.

6. Soza $G$, Vicente $M$, Betancourt $M$, et al.: Bronconeumonía del lactante menor. Correlación clínica, radiológica y etiología viral. Rev Chile Infect 1987; 4; 82-86.

7. Avendario LF, Larratage $C$, Palomino MA, et al.: Communily and hospital-acquired respiratory syncytial virus infection in Chile. Pedialr Infect Dis $\mathbf{J} 1991$; 10: 564-568.

8. Méndez B, Goldenberg E, Vicente $M$, et al.: Diagnóstiso rápido de virus respiratorio sincicial por técnica de inmunofluorescencia en infecciones respiratorias agudas bajas. Pediatría (Santiago) 1987: 30:235-240.

9. Escobar AM, Martinez F, Ceruvi E, et ol.: Etiología de tas infecciones agudas del tracto respiratorio bajo (IRAB) en lactantes hospitalizados: estudios vírologicos. Rey Chil Pediatr 1988; 59: 349.353.

10. Paisley J, Lauer $B, M d n t o s h ~ K$, et at.: Patogens associated with acute lower respiratory tract infections in young children. Pediatr Infecl Dis J 1984; 3 : 14-19.

11. Mclntosh $K_{+}$Chanock $R$ : Respiratory Syncytial Vins. En: Fields BN, Knipe DN, Chanock RM, Melnick JL, Roizman B and Shope RE, eds. Virology. New York: Raven Press, 1990: 1045-1072.

12. Larrañage $C$, Avendaño LF, Gaggero $A$, et al.: Diagnóstico de infección por adenovirus y vinus sincicial 
respiratorio en Jactantes: comparación entre aislamiento e inmunofluorescencia indirecta. Rev Chil Infect 1990; 7: $167-171$.

13. Friedman A, Shella $N$. Arens $M$, e! al.: Value rapid diagnosis of respiratory syncytial virus infection and management of small infants. Clin Pedjatr 1986; 25; 404-406.

14. Papic $Z$, Rodriguez L, Larrañaga $C$, Avendaño $L F$ : Detección de vinus respiratorios en pacientes ambulatorios y hospitalizados. Rev Chil Pediatr 1992; 63: 256261.

15. Boyer $X$ and Cherry $J$ : Non bacterial pneumonia. En: Feigin $\mathrm{K}$. and Cherry J. Textbook of Pediatric Infectious Diseases. Philadelphia: WB Saunders, 1981; 186-196.
16. Simpson W, Hocking PM, Court SDM, et al.: The radiological findings in respiratory syncytial vins infection in children. The correlation of radiological categonies with clinical and virological findings. Pediatr Radiol 1974: 2: 155-160.

17. Hall C. Douglas R: Modes of transmission of RSV. J Pediatr 1981; 99: 100-103.

18. Ericksson M, Forsgren M. Sjoberg S, el al.: Respiratory syncytial viral infection in infant with congenital heart disease. Acta Pediatr Scand 1983; 72: 47-51.

19. Mintz L. Ballard $R$, Sniderman $S$, et al.: Nosocomial respiratory syncylial virus infections in an intensive care nursety: rapid diagnosis by direct immunofluorescence. Pediatr 1979; 64: 149-153. 